\title{
On the Asymptotic Behavior of Neutrinos ${ }^{\dagger}$
}

\author{
Giuseppe Gaetano Luciano ${ }^{1,2}$ (1) \\ 1 Dipartimento di Fisica, Università di Salerno, Via Giovanni Paolo II, 132 I-84084 Fisciano (SA), Italy; \\ gluciano@sa.infn.it \\ 2 Istituto Nazionale di Fisica Nucleare, Sezione di Napoli, Gruppo Collegato di Salerno, Via Giovanni Paolo II, \\ 132 I-84084 Fisciano (SA), Italy \\ + Presented at the 1st Electronic Conference on Universe, 22-28 February 2021; Available online: \\ https://ecu2021.sciforum.net/.
}

\begin{abstract}
Mixing transformations in QFT are non-trivial, since they are connected with the issue of the unitary inequivalence between Fock space for definite flavor fields and Fock space for definite mass fields. This poses the problem of selecting the right (i.e., physical) representation for asymptotic mixed fields. Here, we approach to the study of this inequivalence in the context of mixing of neutrinos. As a test-bench for our investigation, we consider the weak decay of a uniformly accelerated proton within the framework of the minimally extended SM. By relying on some core principles and predictions of the theory, such as the general covariance, the conservation of the family lepton numbers in the tree-level interaction vertices and the CP-symmetry violating effects in neutrino oscillations, we conclude that the only way to keep the formalism internally consistent is by resorting to the flavor representation.
\end{abstract}

Keywords: neutrino mixing; particle decay; Unruh effect; Haag's theorem

Citation: Luciano, G.G. On the Asymptotic Behavior of Neutrinos . Phys. Sci. Forum 2021, 2, 44. https:// doi.org/10.3390/ECU2021-09306

Academic Editor: Herbert Hamber

Published: 22 February 2021

Publisher's Note: MDPI stays neutral with regard to jurisdictional claims in published maps and institutional affiliations.

Copyright: (C) 2021 by the author. Licensee MDPI, Basel, Switzerland. This article is an open access article distributed under the terms and conditions of the Creative Commons Attribution (CC BY) license (https:// creativecommons.org/licenses/by/ $4.0 /)$.

\section{Introduction}

In the context of canonical QFT, Haag's theorem [1] in its modern form [2] states that, given two different representations of the CCR, $\left(H_{1},\left\{\mathcal{O}_{1}^{i}\right\}\right)$ and $\left(H_{2},\left\{\mathcal{O}_{2}^{i}\right\}\right)$ (where $H_{1 / 2}$ are the respective Hilbert spaces and $\left\{\mathcal{O}_{1 / 2}^{i}\right\}$ the sets of related operators in CCR), there exists in general no unitary map $\mathcal{U}$ from $H_{1}$ to $H_{2}$ such that, for each $\mathcal{O}_{1}^{j} \in\left\{\mathcal{O}_{1}^{i}\right\}$, one can write $\mathcal{O}_{2}^{j}=\mathcal{U} \mathcal{O}_{1}^{j} \mathcal{U}^{-1} \in\left\{\mathcal{O}_{2}^{i}\right\}$. The two representations are then said to be unitarily inequivalent (notice that this problem does not arise at all in non-relativistic QM, where Stone-von Neumann uniqueness theorem [3] guarantees that the representations of the CCR are all unitarily equivalent to each other.). Beyond pure mathematical aspects, this theorem features a number of physical phenomena [4], such as the spontaneous symmetry breaking (where the same algebra describes both the normal and symmetry-broken phases) and Hawking black-hole evaporation (for which inequivalent representations of the CCR are associated to different observer's perspectives, the static observer outside the black hole on one hand, the radially free-falling observer on the other).

Recently, the pivotal rôle of inequivalent representations has been highlighted in problems related to the quantization of superpositions of fields with different masses (henceforth, we simply call these superpositions "mixing transformations" and the ensuing fields "mixed" or "flavor fields") [5]. Specifically, it has been shown that mixing transformations at the level of ladder operators exhibit the structure of a rotation nested into a Bogoliubov transformation. As a result, the vacuum for fields with definite flavors becomes a condensate of particle-antiparticle pairs with definite masses, thus giving rise to inequivalent flavor and mass Fock spaces. The question naturally arises as to which of these two representations should actually be regarded as the physical one for asymptotic mixed fields. 
Along this line, a clue to the solution has been provided through the study of weak decay processes involving neutrinos. In particular, consider the inverse $\beta$-decay of a uniformly accelerated proton, which in the frame of the laboratory reads [6]

$$
p \stackrel{a}{\rightarrow} n+e^{+}+v_{e},
$$

where $p, n, e^{+}$and $v_{e}$ denote the proton, neutron, positron and neutrino, respectively, while $a$ stands for the magnitude of the proper acceleration. Aside from our specific goal, we mention that this process also served as a theoretical proof of the necessity of Unruh effect [7] for the general covariance of QFT [8]. Indeed, by switching to the rest-frame of the proton and demanding the scalar decay rate (i.e., the decay rate over the proper time) to be invariant, the conclusion that the proton must now interact with virtual leptons popping out from Unruh thermal bath as

$$
p+e^{-} \rightarrow n+v_{e}, \quad p+\bar{v}_{e} \rightarrow n+e^{+}, \quad p+e^{-}+\bar{v}_{e} \rightarrow n,
$$

is inevitably reached.

The decay (1) was originally addressed in $[8,9]$ in a toy model with massless neutrino. Attempts to embed neutrino mass and mixing were later carried out in $[6,10,11]$ by considering a scenario with only two neutrino flavors. However, conflicting results on the very nature of asymptotic neutrinos were achieved. Following [12], here we analyze the inverse $\beta$-decay in the three-flavor description with neutrino oscillations and CP violation effects. We compute the scalar decay rate in both the laboratory and comoving frames, and compare the final results. By relying on some core principles of the theory, such as the fulfillment of the general covariance and the conservation of the family lepton numbers in the tree-level interaction vertices, we show that the only way to keep the formalism internally consistent is by resorting to the flavor representation.

Throughout the work, we use natural units $k_{B}=\hbar=c=1$ and Minkowski metric with the conventional time-like signature.

\section{Methods}

This section is devoted to set the stage for the computation of the proton decay rate. Following $[6,8,12]$, we describe the neutron $n$ and proton $p$ as excited and unexcited states of a two-level quantum system, the nucleon. In order to account for the uniformly accelerated motion of the proton, we assume that it moves along a Rindler trajectory (as far as the momenta $k_{e}, k_{v}$ of the positron and neutrino are much smaller than the proton and neutron mass, $m_{p}, m_{n}$, one can assume that the emitted neutron keeps on moving along the same Rindler trajectory of the ingoing proton. In what follows, we make use of this approximation without affecting the overall validity of our considerations.).

For accelerations $a$ small enough with respect to the masses of the intermediate bosons $W^{ \pm}$and $Z^{0}$, the interaction action can be described by a semiclassical Fermi-like effective theory, i.e.,

$$
\hat{S}_{I}=\int d^{4} x \sqrt{-g} \hat{J}_{h, \lambda} \hat{J}_{l}^{\lambda}
$$

where $g$ is the determinant of the metric. Here, we have defined the (classic) hadron current as $\hat{J}_{h, \lambda}=\hat{q}(\tau) u_{\lambda} \delta(x) \delta(y) \delta(u-1 / a)$, where $\hat{q}(\tau)=e^{i \hat{H} \tau} \hat{q}(0) e^{-i \hat{H} \tau}$ is the monopole operator, $\hat{H}$ is the nucleon hamiltonian and $G_{F}=|\langle n|\hat{q}(0)| p\rangle|$ is Fermi coupling constant. The four-vector $u^{\lambda}$ represents the nucleon velocity along the Rindler trajectory, which is parameterized by the condition $u=1 / a$, with $u$ being a Rindler spatial coordinate. The nucleon proper time $\tau$ is related to the coordinate time $v$ by $\tau=v / a$. On the other hand, the quantum lepton current reads $\hat{J}_{l}^{\lambda}=\sum_{\ell=e, \mu, \tau}\left(\hat{\Psi}_{v_{\ell}} \gamma^{\lambda} \hat{\Psi}_{\ell}+\hat{\Psi}_{\ell} \gamma^{\lambda} \hat{\Psi}_{v_{\ell}}\right)$, where $\hat{\Psi}_{\ell\left(v_{\ell}\right)}$ is the electron (neutrino) Dirac field of flavor $\ell$.

A remark is now in order. According to Pontecorvo's pioneering works on two-flavor mixing $[13,14]$ and their extension to three generations [15], it is a well-established fact 
that neutrinos weakly interact with charged leptons in flavor eigenstates $\left|v_{\ell}\right\rangle$, which are superpositions of mass states $\left|v_{j}\right\rangle(j=1,2,3)$ via PMNS matrix $U \equiv U\left(\theta_{12}, \theta_{23}, \theta_{13}, \delta\right)$, where $\theta_{12}, \theta_{23}$ and $\theta_{13}$ are the three mixing angles and $\delta$ the CP violating phase (see [12] for the explicit expression of $U$ ). Notice that this is mandatory to obey the conservation of the family lepton numbers in the (tree-level) interaction vertices. In the next Section, we shall see how to take advantage of this feature in exploring the flavor-mass controversy.

\section{Results and Discussion}

Let us now give computational details. Firstly, we analyze the inverse $\beta$-decay in the laboratory frame, where the accelerating source provides the proton with the missing energy to convert into a neutron, a positron and an electron neutrino according to (1). We then study the process from the point of view of an observer comoving with the proton (see (2)). As commented above, in this case the proton at rest is allowed to decay due to the interaction with electrons and antineutrinos in Unruh thermal bath.

\subsection{Laboratory Frame}

By making use of the $S$-matrix formalism, the (tree-level) scalar decay rate for the process (1) takes the form [12]

$$
\begin{aligned}
\Gamma \equiv & \frac{1}{T} \sum_{\sigma_{e}, \sigma_{v}} \int d^{3} k_{v} \int d^{3} k_{e}|\mathcal{A}|^{2} \\
= & \left|U_{e 1}\right|^{4} \Gamma_{1}+\left|U_{e 2}\right|^{4} \Gamma_{2}+\left|U_{e 3}\right|^{4} \Gamma_{3} \\
& +\left(\left|U_{e 1}\right|^{2}\left|U_{e 2}\right|^{2} \Gamma_{12}+\left|U_{e 1}\right|^{2}\left|U_{e 3}\right|^{2} \Gamma_{13}+\left|U_{e 2}\right|^{2}\left|U_{e 3}\right|^{2} \Gamma_{23}+\text { c.c. }\right),
\end{aligned}
$$

where $\sigma_{e(v)}$ is the electron (neutrino) polarization, $T$ is the nucleon proper time and we have denoted by $\mathcal{A}$ the transition amplitude $\mathcal{A}=\left\langle n\left|\otimes\left\langle e^{+}, v_{e}\left|\hat{S}_{I}\right| 0\right\rangle \otimes\right| p\right\rangle . U_{\ell, i}(\ell=\{e, \mu, \tau\}, i=$ $\{1,2,3\})$ represents the generic element of PMNS matrix, while the explicit expressions of $\Gamma_{i}$ and $\Gamma_{i j}$ are given in Equations (19) and (20) of [12].

At this stage, we notice that, due to the asymptotic occurrence of flavor oscillations, the total decay rate also gets non-trivial contributions from the processes $p \stackrel{a}{\rightarrow} n+e^{+}+v_{\mu(\tau)}$. By adding up the three rates, in the laboratory frame we finally obtain [12]

$$
\Gamma^{\mathrm{lab}}=\left|U_{e 1}\right|^{2} \Gamma_{1}+\left|U_{e 2}\right|^{2} \Gamma_{2}+\left|U_{e 3}\right|^{2} \Gamma_{3} .
$$

\subsection{Comoving Frame}

In the comoving frame, the evaluation of the decay rate is contingent upon the quantization of lepton fields in the Rindler-Fulling scheme, which is the procedure of quantization pertaining to a uniformly accelerated observer in Minkowski spacetime. Bearing in mind that the proton can absorb (emit) a particle of Rindler frequency $\omega$ from (toward) the thermal bath with probability $n_{F}(\omega)=\left(e^{\omega / T_{U}}+1\right)^{-1}\left(\widetilde{n}_{F}(\omega)=1-n_{F}(\omega)\right)$, where $T_{\mathrm{U}}=a / 2 \pi$ is Unruh temperature [7], the decay rate for the three process (2) is

$$
\begin{aligned}
\Gamma= & \left|U_{e 1}\right|^{4} \widetilde{\Gamma}_{1}+\left|U_{e 2}\right|^{4} \widetilde{\Gamma}_{2}+\left|U_{e 3}\right|^{4} \widetilde{\Gamma}_{3} \\
& +\left(\left|U_{e 1}\right|^{2}\left|U_{e 2}\right|^{2} \widetilde{\Gamma}_{12}+\left|U_{e 1}\right|^{2}\left|U_{e 3}\right|^{2} \widetilde{\Gamma}_{13}+\left|U_{e 2}\right|^{2}\left|U_{e 3}\right|^{2} \widetilde{\Gamma}_{23}+\text { c.c. }\right),
\end{aligned}
$$

where $\widetilde{\Gamma}_{i}$ and $\widetilde{\Gamma}_{i j}$ are defined as in Equations (39) and (40) of [12].

Once again, the necessity to account for flavor oscillations requires us to consider also the following extra channels: $p+e^{-} \rightarrow n+v_{\mu(\tau)}, p+\bar{v}_{\mu(\tau)} \rightarrow n+e^{+}$and $p+e^{-}+\bar{v}_{\mu(\tau)} \rightarrow n$. Therefore, the total decay rate in the comoving frame becomes [12]

$$
\Gamma^{\mathrm{com}}=\left|U_{e 1}\right|^{2} \widetilde{\Gamma}_{1}+\left|U_{e 2}\right|^{2} \widetilde{\Gamma}_{2}+\left|U_{e 3}\right|^{2} \widetilde{\Gamma}_{3}
$$


Clearly, since we are dealing with a scalar quantity, we expect that $\Gamma^{\mathrm{lab}}=\Gamma^{\mathrm{com}}$. This has been explicitly proved in [6,12] (at least in the realistic approximation of small neutrino mass differences), showing that $\Gamma_{i}=\widetilde{\Gamma}_{i}, \Gamma_{i j}=\widetilde{\Gamma}_{i j}$, for all $i, j$. We thus find that the use of flavor states is consistent with the general covariance of the theory. Actually, we point out that the same conclusion would be reached by working in the mass representation [11], forcing us to look for some other criterion to discern between flavor and mass states.

In this vein, let us consider as a test-bench the description of $\mathrm{CP}$ symmetry violating effects in neutrino oscillations, which are predicted by the SM and have also been measured using long-baseline neutrino and antineutrino oscillations observed by the T2K experiment (see [16] and references therein). Such effects can be quantified by introducing the so-called Jarlskog invariant $J$, which is defined by $\operatorname{Im}\left[U_{\delta i} U_{\gamma_{i}}^{*} U_{\delta j}^{*} U_{\gamma j}\right] \equiv J \sum_{\lambda, k} \varepsilon_{\delta \gamma \lambda} \varepsilon_{i j k}$, where $\delta, \gamma, \lambda=\{e, \mu, \tau\}$ and $i, j, k=\{1,2,3\}$. Obviously, since this is the unique phaseindependent measure of $\mathrm{CP}$ violation that we can build from PMNS matrix, all CP violating observables are expected to depend on it.

In order to feature the flavor-mass dichotomy via the study of CP violation, we consider the scattering matrix $\hat{S}_{\text {weak }}$ for a generic charged-current weak interaction with an outgoing neutrino (the specific case of the inverse $\beta$-decay is recovered by simply equating $\hat{S}_{\text {weak }}$ to $\hat{S}_{I}$ given in Equation (3)). To quantify CP violation, we assume that the neutrino, emitted for instance with flavor $e$, undergoes oscillation and is detected after a certain distance with flavor $\mu$. By working in the asymptotic flavor basis, the transition amplitude for this process is

$$
\mathcal{A}_{v_{e}, v_{\mu}}={ }_{\text {out }}\left\langle v_{\mu}, \ldots\left|S_{\text {weak }}\left(\bar{\psi}_{v_{e}} \ldots\right)\right| \ldots\right\rangle_{\text {in }},
$$

where the dotted spaces must be filled with the other fields/states involved in the interaction. After implementing the mixing transformation on both the neutrino field and state, we are led to the following expression for the decay rate

$$
\begin{aligned}
& \Gamma_{v_{e}, v_{\mu}} \sim\left|\mathcal{A}_{v_{e}, v_{\mu}}\right|^{2}=\left|U_{\mu 1}\right|^{2}\left|U_{e 1}\right|^{2}\left|\mathcal{A}_{1}\right|^{2}+\left|U_{\mu 2}\right|^{2}\left|U_{e 2}\right|^{2}\left|\mathcal{A}_{2}\right|^{2}+\left|U_{\mu 3}\right|^{2}\left|U_{e 3}\right|^{2}\left|\mathcal{A}_{3}\right|^{2} \\
& +\left(U_{\mu 1}^{*} U_{e 1} U_{\mu 2} U_{e 2}^{*} \mathcal{A}_{1} \mathcal{A}_{2}^{*}+U_{\mu 1}^{*} U_{e 1} U_{\mu 3} U_{e 3}^{*} \mathcal{A}_{1} \mathcal{A}_{3}^{*}+U_{\mu 2}^{*} U_{e 2} U_{\mu 3} U_{e 3}^{*} \mathcal{A}_{2} \mathcal{A}_{3}^{*}+\text { c.c. }\right),
\end{aligned}
$$

where we have omitted the sum over polarizations and the integration over momenta to streamline the notation.

Let us now focus on the mirror-symmetric interaction (Parity transformation) and swap particles with antiparticles (Charge conjugation). By evaluating the decay rate $\Gamma_{\bar{\nu}_{e}, \bar{\nu}_{\mu}}$ for this process, we can finally quantify $\mathrm{CP}$ asymmetry as

$$
A_{C P}^{(e, \mu)} \equiv \Gamma_{v_{e}, v_{\mu}}-\Gamma_{\bar{v}_{e}, \bar{\nu}_{\mu}}=4 J\left\{-\operatorname{Im}\left[\mathcal{A}_{1} \mathcal{A}_{2}^{*}\right]+\operatorname{Im}\left[\mathcal{A}_{1} \mathcal{A}_{3}^{*}\right]-\operatorname{Im}\left[\mathcal{A}_{2} \mathcal{A}_{3}^{*}\right]\right\} .
$$

As expected, this quantity is non-vanishing and proportional to the Jarlskog invariant $J$. On the other hand, it is quite unclear how to reproduce such a result if working with asymptotic mass states. In fact, as shown in [12], in that case, one would trivially get $A_{C P}=0$, hinting that the mass representation is inconsistent with the prediction of $\mathrm{CP}$ violation in neutrino oscillations.

\section{Conclusions}

Understanding the very nature of asymptotic neutrinos is a challenging, but fundamental task, given their abundance in the universe and their unparalleled rôle in investigating physics at all energy scales [17]. Waiting for experimental hints, in this work we have approached this study from a purely theoretical perspective. By analyzing the weak decay of a uniformly accelerated proton within the framework of the SM, we have shown that a generally covariant formalism consistent with (i) the conservation of the family lepton numbers in the interaction vertices, (ii) the phenomenologically observed neutrino oscillations 
and (iii) the related CP symmetry violation can be formulated, provided that asymptotic neutrinos are described through flavor states.

Clearly, further questions need to be answered. First, we have developed computations up to the leading order in neutrino mass differences. To strengthen our claim, the exact calculation should be performed. Nevertheless, we expect that higher order terms do not spoil the overall validity of our result, although some novel effects might appear. For instance, in [18], it has been argued that Unruh distribution for mixed fields acquires extra terms that break down its thermal nature. It would then be interesting to explore the interplay of this exotic behavior of Unruh effect with our findings. Special focus is also deserved by the study of inertial effects on the oscillation probability formula, given their intimate connection with gravity-induced corrections. More work is inevitably required in these and other directions.

Funding: This research received no external funding.

Institutional Review Board Statement: Not applicable.

Informed Consent Statement: Not applicable.

Data Availability Statement: Not applicable.

Conflicts of Interest: The author declares no conflict of interest.

\begin{tabular}{|c|c|}
\hline Abbre & ations \\
\hline The $f$ & wing abbreviations are used in this $n$ \\
\hline QFT & Quantum Field Theory \\
\hline SM & Standard Model \\
\hline $\mathrm{CP}$ & Charge Conjugation \& Parity \\
\hline CCR & Canonical Commutation Relations \\
\hline QM & Quantum Mechanics \\
\hline PMNS & Pontecorvo-Maki-Nakagawa-Sakata \\
\hline
\end{tabular}

\section{References}

1. Haag, R. On quantum field theories. Mat.-Fys. Medd. 1955, 29, 12.

2. Earman, J.; Fraser, D. Haag's Theorem and Its Implications for the Foundations of Quantum Field Theory. Erkenntnis 2006, 64, 305. [CrossRef]

3. von Neumann, J. Die Eindeutigkeit der Schrödingerschen Operatoren. Ann. Math. 1931, 104, 570. [CrossRef]

4. Blasone, M.; Jizba, P.; Smaldone, L. Functional integrals and inequivalent representations in Quantum Field Theory. Ann. Phys. 2017, 383, 207. [CrossRef]

5. Blasone, M.; Vitiello, G. Quantum field theory of fermion mixing. Ann. Phys. 1995, 244, 283. [CrossRef]

6. Blasone, M.; Lambiase, G.; Luciano, G.G.; Petruzziello, L. Role of neutrino mixing in accelerated proton decay. Phys. Rev. D 2018, 97, 105008. [CrossRef]

7. Unruh, W.G. Notes on black-hole evaporation. Phys. Rev. D 1976, 14, 870. [CrossRef]

8. Vanzella, D.A.T.; Matsas, G.E.A. Decay of accelerated protons and the existence of the Fulling-Davies-Unruh effect. Phys. Rev. Lett. 2001, 87, 151301. [CrossRef] [PubMed]

9. Muller, R. Decay of accelerated particles. Phys. Rev. D 1997, 56, 953. [CrossRef]

10. Ahluwalia, D.V.; Labun, L.; Torrieri, G. Neutrino mixing in accelerated proton decays. Eur. Phys. J. A 2016, 52, 189. [CrossRef]

11. Cozzella, G.; Fulling, S.A. L.; Ulfo, A.G.S.; Matsas, G.E.A.; Vanzella, D.A.T. Unruh effect for mixing neutrinos. Phys. Rev. D 2018, 97, 105022. [CrossRef]

12. Blasone, M.; Lambiase, G.; Luciano, G.G.; Petruzziello, L. On the $\beta$-decay of the accelerated proton and neutrino oscillations: A three-flavor description with CP violation. Eur. Phys. J. C 2020, 80, 130. [CrossRef]

13. Pontecorvo, B. Mesonium and anti-mesonium. Sov. Phys. JETP 1957, 6, 429.

14. Pontecorvo, B. Neutrino Experiments and the Problem of Conservation of Leptonic Charge. Sov. Phys. JETP 1968, $26,984$.

15. Maki, Z.; Nakagawa, M.; Sakata, S. Remarks on the unified model of elementary particles. Prog. Theor. Phys. 1962, 28, 870. [CrossRef]

16. Abe, K.; Akutsu, R.; Ali, A.; Alt, C.; Andreopoulos, C.; Anthony, L.; Antonova, M.; Aoki, S.; Ariga, A.; Asada, Y.; et al. Constraint on the matter-antimatter symmetry-violating phase in neutrino oscillations. Nature 2020, 580, 339; Erratum in: Nature 2020, 583 , E16. 
17. Luciano, G.G.; Petruzziello, L. Testing gravity with neutrinos: From classical to quantum regime. Int. J. Mod. Phys. D 2020, 29, 2043002. [CrossRef]

18. Blasone, M.; Lambiase, G.; Luciano, G.G. Nonthermal signature of the Unruh effect in field mixing. Phys. Rev. D 2017, $96,025023$. [CrossRef] 